\title{
Structural Investigations on Poly(methyl methacrylate) Various Composites Used for Stereolithographyc Complete Dentures
}

\author{
EUGENIA EFTIMIE TOTU ${ }^{1}$, CORINA MARILENA CRISTACHE ${ }^{2}$, SELIM ISILDAK ${ }^{3}$, OZLEM TAVUKCUOGLU ${ }^{3}$, AIDA PANTAZI ${ }^{4}$, \\ MARIUS ENACHESCU ${ }^{4}$, ROXANA BUGA ${ }^{5}$, MIHAI BURLIBASA ${ }^{2}$, TIBERIU TOTU 5 \\ IUniversity Politehnica of Bucharest, Faculty of Applied Chemistry and Material Science, 1-5 Polizu Str, 11061 Bucharest, \\ Romania. \\ 2University of Medicine and Pharmacy Carol Davila, Faculty of Midwifery and Medical Assisting (FMAM), Department of Dental \\ Techniques, 8, Eroilor Sanitari Blvd, 050474, Bucharest, Romania \\ ${ }^{3}$ Department of Bioengineering, Faculty of Chemical and Metallurgical Engineering,Yildiz Technical University, 34210 Esenler- \\ Istanbul, Turkey \\ ${ }^{4}$ University Politehnica of Bucharest, Center for Surface Science and Nanotechnology (CSSNT), 313 Splaiul Independentei, \\ 060042, Bucharest, Romania \\ ${ }^{5}$ University Politehnica of Bucharest,Faculty of Electronics and Telecommunications,313 Spl. Independentei, 060042, Bucharest, \\ Romania
}

\begin{abstract}
The present paper is focused on analyzing if appropriate adhesion between the polymeric matrix and titania filler nanoparticles is obtained for the PMMA-TiO photo-curable dental material, suitable for application in $R P$ - stereolithography (SLA) for complete denture manufacturing. It was found that different amounts, between $0.2 \%$ and $2.5 \%$ (w/w\%), of added titanium oxide nanoparticles slightly modify the structural behavior of the PMMA polymeric matrix. The material characterization was carried out using FT-IR and microscopy techniques.
\end{abstract}

Keywords: PMMA - TiO ${ }_{2}$ functionalized nanocomposite, structural analysis, stereolithography, complete denture

The advancements in computer-aided design and computer aided manufacturing (CAD/CAM) encouraged its applications in fixed and removable prosthodontics, leading to the development of new technologies and new materials with improved biomechanical characteristics and high biocompatibility. To date, subtractive technology (milling from gingiva-colored pre-polymerized acrylic blank) for denture base and prefabricated teeth are mostly used, in clinical environment, to manufacture CAD/CAM dentures. Despite of the important advantages of this technique, such as reduced number of appointments, rapid fabrication, improved fit and electronic archiving of prosthetic design, computer engineered complete dentures have some important drawbacks, mainly regarding the expensive materials and increased laboratory costs [1,2]. Additive manufacturing or rapid prototyping (RP) and the use of improved polymers has been foreseen as a viable and less costly solution for complete denture manufacturing. Furthermore, if a monolithic denture/ overdenture $[3,4]$ is envisaged, the functionalization of the polymeric teeth during the design may also be performed [5]. Poly (methyl methacrylate) (PMMA), the material of choice for removable dentures, has been lately investigated and there are studies highlighting the effect of adding nanofillers such as titanium dioxide $\left(\mathrm{TiO}_{2}\right)$ to improve their specific properties [6]. PMMA-nano $\mathrm{TiO}_{2}$ composite have shown better thermal behavior [7] in connection with a lower water sorption and solubility [8]. From the mechanical point of view, such composites are characterized by an improved hardness, fracture resistance and/or flexural strength [9]. However, some studies have shown lower flexural strength when titania nanoparticles were added into a poly (methyl methacrylate) matrix [10]. Such noted behavior could be the result of a poor homogeneity of the final material.
The present study was focused on obtaining an appropriate adhesion between the polymeric matrix and titania filler nanoparticles for the PMMA-TiO photopolymerizing material for dental use, important for enhancing the nanocomposite's characteristics. The structural properties of the PMMA-nanocomposites suitable for application in RP - stereolithography (SLA) dentistry have been characterized by FT-IR analysis and microscopy investigations.

\section{Experimental part}

Reagents and apparatus

The reagents used in this work are similar to those presented in our previously published paper [7]. The polymer matrix, denoted as PMMA-D, consisting in a mixture of poly(methyl metharcrylate)(PMMA) -methyl methacylate (MMA) and reinforcing additives solution (from EnvisionTEC GmbH, Gladbeck, Germany) has been used throughout experiments, as provided. The nanofiller added to the polymeric matrix was the titanium oxide (anatase type) - from Aldrich. The obtained material has been used for complete dentures manufacturing employing STL technology. The applied workflow, described elsewhere $[11,12]$, was run on Envison TEC equipment.

In order to avoid the inhomogeneity and local agglomeration of the nanofiller within the PMMA matrix, the titania nanoparticles have been functionalized with methacrylic acid (Aldrich) according to the procedure described in detail in [7]. After functionalization, the nanofiller was carefully mixed with the PMMA-D matrix. The necessary amounts of the two components of the nanocomposite were precisely weighed with an analytical balance in order to obtain a concentration between 0.2 and $2.5(\mathrm{w} / \mathrm{w})$ for $\mathrm{TiO}_{2}$ nanoparticles in polymer. 
The SEM morphologies of the prepared nanocomposites have been studied using a scanning electron microscopy (SEM) system, from Oxford Instruments. In addition, the elemental mapping was obtained through EDX analysis performed on the same equipment. Other microscopy investigations carried on a bright field microscope were performed using Leica DM 3000 LED device, equipped with an MC 190 HD camera based on s-CMOS sensor with reduced noise factor.

The subsequent structural analysis was investigated by Attenuated Total Reflectance Fourier-Transformed Infrared spectroscopy (ATR-FTIR). The FTIR spectra of all samples were recorded in the $4000-500 \mathrm{~cm}^{-1}$ range, with a resolution of $4 \mathrm{~cm}^{-1}$ at room temperature using a Bruker Tensor 27 equipment (diamond ATR). A polymeric matrix without titanium oxide nanoparticles was used as a reference sample. Raman studies, which are complementary with FT-IR measurements, were performed by Confocal Micro Raman Spectroscopy using a LabRam HR800 system equipped with a green He-Ne laser (532 nm) as the excitation source. All the Raman spectra were collected by exposing the specimens during $100 \mathrm{~s}$, in the $3600-100$ $\mathrm{cm}^{-1}$ region with a spectral resolution of $0.6 \mathrm{~cm}^{-1}$. All the experiments were performed at room temperature.

\section{Results and discussions}

An important aspect for the good functioning of composite materials, such as PMMA - TiO, is the filler's dispersion in the polymeric matrix. Therefore, the resulted morphology is further influencing the material characteristics. Taking advantage of microscopy techniques, it is possible to investigate, atmicroscopic level, the PMMA - $\mathrm{TiO}_{2}$ nanocomposites' structure.

The microscopy analysis confirmed a satisfactory dispersion of TiO2 particles in PMMA polymer matrix. The degree of nanoparticles dispersion is conditioned by the amount of filler added into the polymer (fig. 1).
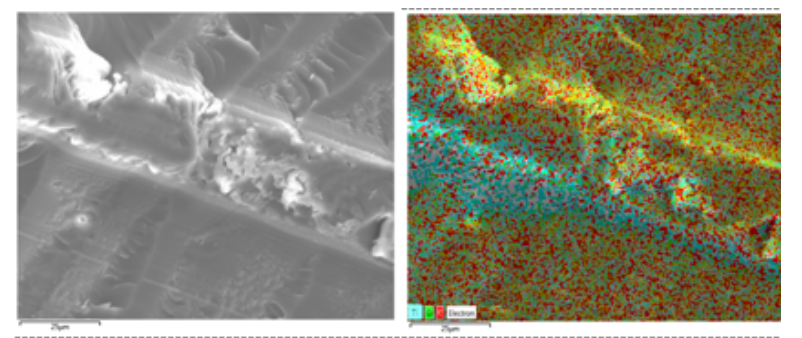

a.

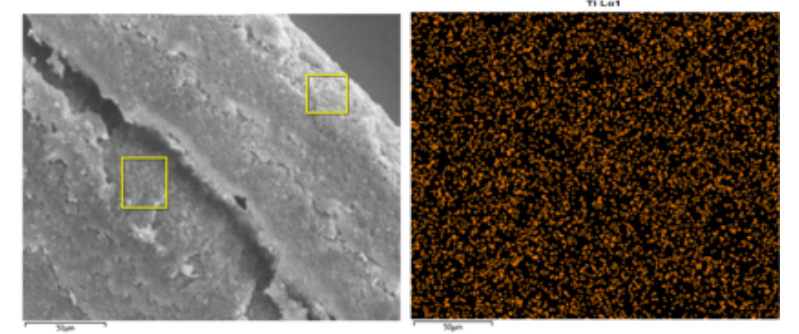

Fig. 1. SEM images with corresponding EDX elemental maps

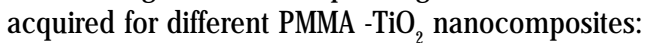
a. PMMA $-0.4 \% \mathrm{TiO}_{2}$; b. PMMA - $1 \% \mathrm{TiO}_{2}$.

The nanocomposite with lower concentration of titania nanoparticles is more homogeneous. Therefore, it could be concluded that PMMA polymer and $\mathrm{TiO}_{2}$ nanoparticles are compatible, as no exudation process has been observed. For the composite with only $0.2 \%$ added $\mathrm{TiO}_{2}$ no phase separation has been observed (fig. 2).

A higher amount of the added nanofiller added lead to the formation of randomly distributed clusters [1] , shown in Figure 3. As it can be observed, the surface roughness is

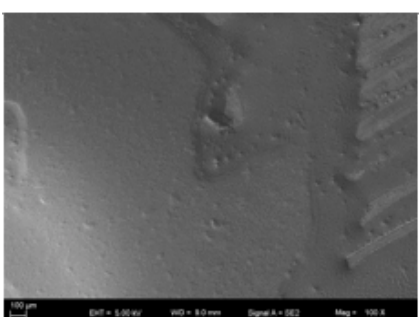

a.

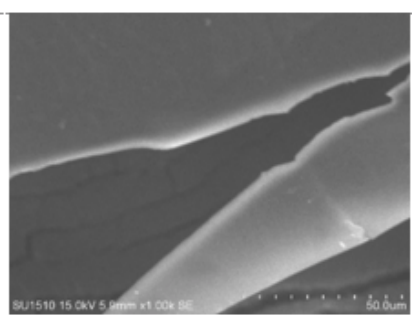

b.
Fig. 2. SEM Images for: a. PMMA and b. PMMA - $0.2 \% \mathrm{TiO}_{2}$ nanocomposite

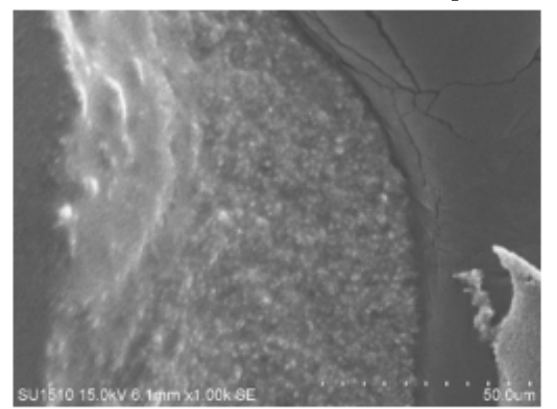

Fig. 3. SEM image

showing cluster formation for PMMA $2.5 \% \mathrm{TiO}_{2}$ nanocomposite

significantly modified with the increasing of $\mathrm{TiO}_{2}$ nanoparticles ratio. The increase of the surface roughness is most likely caused by the aggregation $\mathrm{TiO}_{2}$ nanoparticles into the polymeric host.

The image presented in figure 4 , obtained with the bright field microscope, is showing the dispersion of the metallic nanofiller in the PMMA matrix, revealing the existence of a continuous media without considerable particle conglomerates.

The influence of the added amount of $\mathrm{TiO}_{3}$ nanoparticles into the PMMA polymer is also evidenced by the FT-IR spectra illustrated in figures 5 and 6.



From these spectra, it should be noted that as a result of the incorporation of different titania quantities into the matrix three important effects are observed: the intensity of the absorption band due to the filler presence increases; the maxima positions of the main absorption bands are shifted towards higher wavenumbers; the profile of the absorption band has changed. The shift of the absorption band is illustrated through inserts on figure 7.

Figure 5 presents the FT-IR spectrum of PMMA matrix without any titania nanoparticles. The specific absorption 


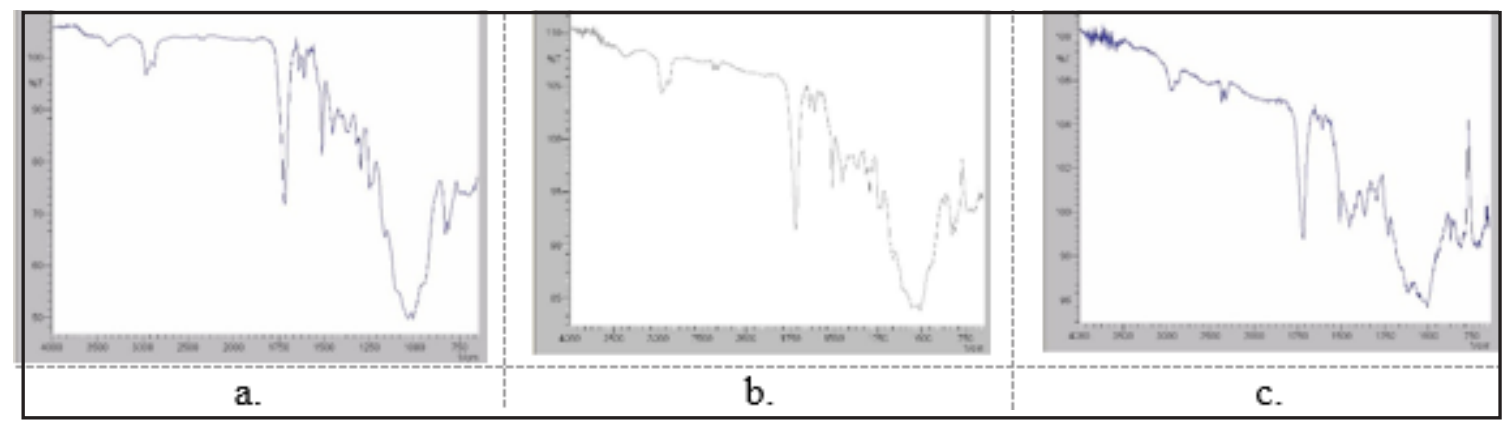

Fig. 6. FT-IR spectra for: a. PMMA- 0.4\% TiO nanocomposite; b. PMMA- $1 \% \mathrm{TiO}_{2}$ nanocomposite; c. PMMA- $2.5 \% \mathrm{TiO}_{2}$ nanocomposite

bands from $1384 \mathrm{~cm}^{-1}, 1031 \mathrm{~cm}^{-1}, 828 \mathrm{~cm}^{-1}$ and $747 \mathrm{~cm}^{-1}$, are characteristic for the polymeric matrix to be used for stereolithographic technique [6]. The initial spectral curves were recorded before exposing the samples to UV radiation specific for the employed AM technology. The FT-IR spectra presented for samples with different amounts of titania nanofiller put in evidence that curve recorded for the sample with $0.2 \%$ nanofiller concentration is not significantly modified compared to the reference spectrum - PMMA without any nanoparticles added.

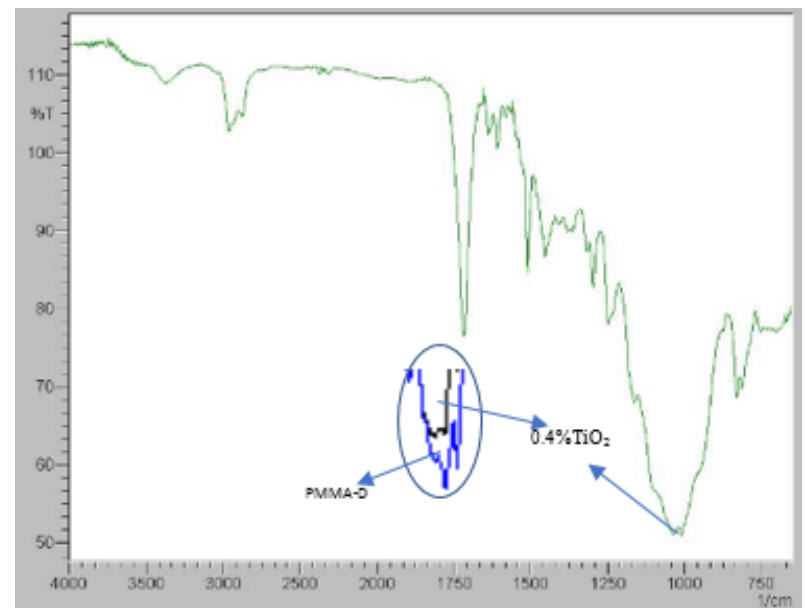

Fig. 7. FT-IR spectrum for UV exposed PMMA - 0.4\% $\mathrm{TiO}_{2}$ nanocomposite

It could be observed that there are two weak peaks at $3384 \mathrm{~cm}^{-1}$ and $609 \mathrm{~cm}^{-1}$ specific for $-\mathrm{OH}$ group stretching and bending vibrations, respectively. The -OH groups most probably come from the adsorbed water molecules. The $\mathrm{C}-\mathrm{H}$ bond (from $-\mathrm{CH}_{3}$ and $-\mathrm{CH}_{2}$ groups) stretching vibrations bands are placed at about $2960 \mathrm{~cm}^{-1}$. The specific vibration band for $\mathrm{C}=0$ stretching of acrylate carboxyl group is present at $1719 \mathrm{~cm}^{-1}$, while at $1453 \mathrm{~cm}^{-1}$ there is a vibration band that could be attributed to the bending vibration of the $\mathrm{C}-\mathrm{H}$ bonds from $-\mathrm{CH}_{3}$ group. The bands at 1384 and $747 \mathrm{~cm}^{-1}$ can be attributed to the $\alpha$-methyl group vibrations. The bands known as the characteristic absorption vibrations of PMMA are present at $1031 \mathrm{~cm}^{-1}$ and $828 \mathrm{~cm}^{-1}$.
For instance, there were no important differences found between FTIR spectrum of PMMA with \%0.4 TiO and PMMA with \%0.6 TiO. However, the intensities of almost all the peaks exhibitêd by the nanocomposite with $\% 0.4$ TiO are lower.

The results shown in the inset of figure 7 put in evidence that the shift of the absorption band depends on the amount of the added nanofiller in the case of UV exposed specimens, as well. Specifically, for the $0.4 \%$ TiO $-P M M A$ composite it was about $20 \mathrm{~cm}^{-1}$. The shift increased afterwards, reaching about $50 \mathrm{~cm}^{-1}$ for $2.5 \% \mathrm{TiO}$ added.

Figure 7 presents the FT-IR spectrum of PMMA - TiO, nanocomposite after UV exposure. It could be easily noticed that, in this case, the FT-IR spectrum of the sample is different from those obtained for the un-irradiated samples.

The specific absorption band for $\mathrm{TiO}_{2}$ in connection with the stretching vibrations of bridging oxygen atoms is present within the wavenumber range $550-900 \mathrm{~cm}^{-1}$, and the position of the peak depends on the structural arrangement of the oxygen atoms [13].

It is well known that $\mathrm{TiO}_{2}$ have specific Raman fingerprints for both, anatase or rutile, structures. The anatase-type titania is characterized by the tetragonal space group $\mathrm{D}_{4 \mathrm{th}}$ (14/amd, with $\mathrm{Z}=4$ for anatase), while the rutile structure belongs to the $D_{4 h}(P 4 / \mathrm{mnm}$, with $Z=$ 2 for rutile) tetragonal space group $[14,15]$. Anatase is characterized by six Raman active modes $\left(1 \mathrm{~A}_{1}+2 \mathrm{~B}_{1}+\right.$ $3 E_{\text {) }}$, while rutile possesses four Raman active modes ( $B$ $\mathrm{E}^{\prime}{ }^{9} \mathrm{~A}_{1,}$, and $\left.\mathrm{B}_{2}\right)$. The Raman spectra illustrated in Figure 8 confirm the successful incorporation of titania nanoparticles in the PMMA-TiO nanocomposite. Also, it could be observed that the $\mathrm{TiO}_{\text {, nanoparticles are present }}$ in both phases, anatase and rutile.

The Raman frequencies for the PMMA sample with $0.4 \%$ TiO are $141 \mathrm{~cm}^{-1}, 196 \mathrm{~cm}^{-1}, 398 \mathrm{~cm}^{-1}, 514 \mathrm{~cm}^{-1}, 517 \mathrm{~cm}^{-1}$ and $636 \mathrm{~cm}^{-1}$ for anatase, while $392 \mathrm{~cm}^{-1}$ and $805 \mathrm{~cm}^{-1}$ for the $\mathrm{TiO}_{2}$ rutile phase.

The Raman spectra recorded for the initial matrix without titania nanofiller and for the PMMA matrix with $0.4 \% \mathrm{TiO}_{2}$ nanoparticles after their exposure to UV radiation

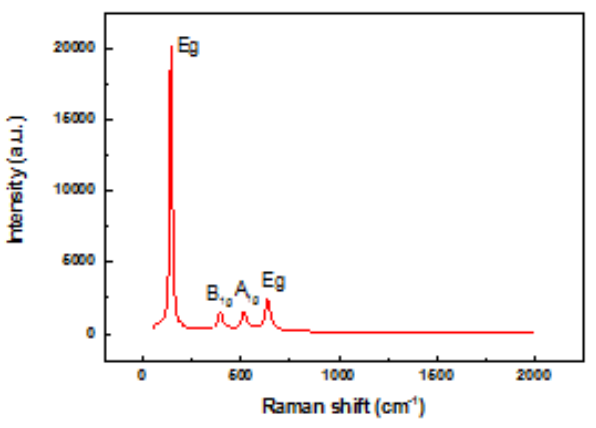

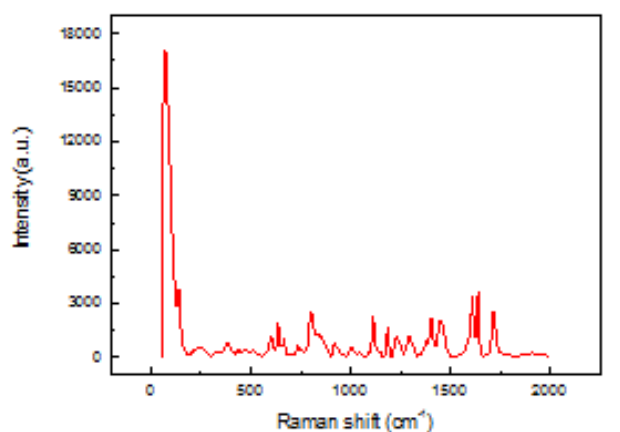

Fig. 8. Raman spectra. a. TiO nanoparticles; b. composite PMMA + $0.4 \% \mathrm{TiO}_{2}$ nanoparticles 


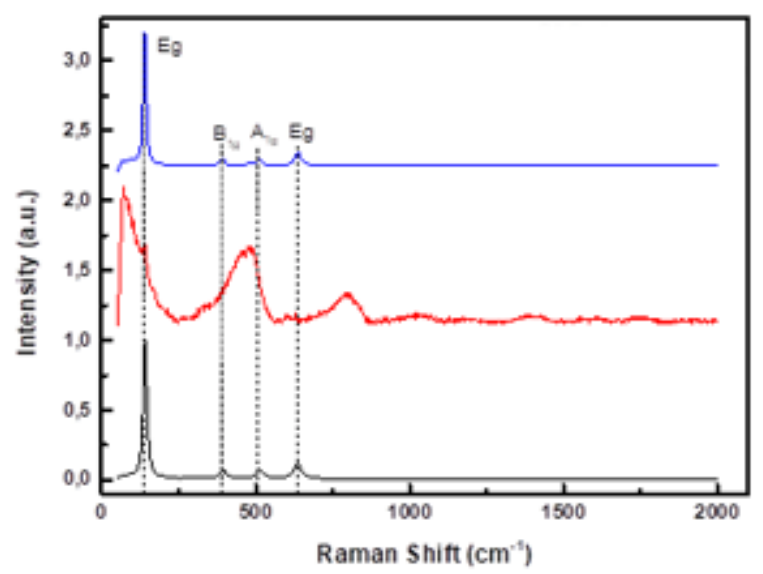

Fig. 9. Raman spectrum of: $\mathrm{TiO}_{2}$ nanoparticles (black line), UV irradiated PMMA (red line) and UV irradiated PMMA $+0.4 \% \mathrm{TiO}_{2}$ nanoparticles composite (blue line)

are shown in figure 9 . In addition, the specific Raman shifts for titania nanoparticles have been recorded and presented on the same graph.

The polymeric materials selected for complete dental prostheses need to have similar physical, mechanical, and aesthetic characteristics to natural teeth. It is well known that for edentulous patients, removable PMMA dentures and overdentures are used to overcome the loss of all teeth [16]. Nevertheless, the usual mechanical failure of the PMMA denture base and the acrylic teeth, susceptible to abrasion could lead to a great number of complications. Dealing with complications is not only an expensive matter but is also inconvenient for the patients. Lately, several studies [7,17] proved that doping the PMMA with an appropriate filler, as $\mathrm{TiO}_{2}$ nanoparticles could modify the specific properties of polymeric matrix resulting into a composite material with improved functions.

The new trends in the additive dental technologies, i.e. $\mathrm{RP}$ technique, will redirect the dental materials research towards complex nanocomposites.

\section{Conclusions}

The morphological and structural studies presented in this work offer a valuable confirmation of our previous findings $[6,7,18]$ regarding the improved characteristics of the PMMA-TiO 2 nanocomposite.

In the light of the new emerging RP technology, the PMMA-TiO , nanocomposite that we are proposing could be considered as a promising 3D printing material for complete dentures and overdentures manufacturing.
Acknowledgement: This work was supported by a grant of the Romanian National Authority for Scientific Research and Innovation, CCCDI - UEFISCDI, project number 30/2016-PRIDENTPRO, (ERA-NETMANUNET II) within PNCDI III. Also, this research was partially supported by the scýentific and technological research council of Turkey, TUBITAK, project number 9150165, MANUNET ERA-NET.

\section{References}

1.BIDRA, A., S., TAYLOR, T., D., AGAR, J., R., J. Prosthet. Dent., 109, 2013, p. 361.

2.BILGIN, M., S., BAYTAROGLU, E., N., ERDEM, A., DILBER, E., Eur. J. Dent., vol.10, 2, 2016, p.286.

3.CRISTACHE, C., M., IONESCU, C., CRISTACHE, G., IONESCU, I., ILIESCU, A., A., BURLIBASA, M., Metal. Int. 14, (SPEC. ISS.16), 2009, p.27.

4.CRISTACHE, C., M., IONESCU, C., BURLIBASA, M., CRISTACHE, G., ILIESCU, A. A., DUMITRIU, H., T., Metal. Int. 14, (SPEC. ISS.16), 2009, p.59.

5.OANCEA, L., STEGAROIU, R., CRISTACHE, C., M., Ann. Anat., 218, 2018, p.49.

6.TOTU, E. E., NECHIFOR, A. C., NECHIFOR, G., ABOUL-ENEIN, H. Y., CRISTACHE, C. M., J. Dent., 59, 2017, p. 68.

7.TOTU, E. E., CRISTACHE, C. M., VOICILA, E., OPREA, O., AGIR, I., TAVUKCUOGLU, O., DIDILESCU, A. C., Mat. Plast., 54, no. 4, 2017, p.666.

8.AHMED, M., A., EL-SHENNAWY, M., ALTHOMALI, Y., M., OMAR, A., A., World J Nano Sci Eng., 6, 2016, p.111.

9.NEJ ATIAN, T., JOHNSON, A., NOORT, R., V., Adv Sci Technol., 49, 2006, p.124.

10.ASAR, N., V, ALBAYRAK, H., KORKMAZ, T., TURKYILMAZ, I., J Adv Prosthodont., 5, 2013, p.241

11.CRISTACHE, C., M., TOTU, E., E., MOCANU, G., BUTNARASU, C., dentalTarget., vol. XI, no. 3(4), 2016, p. 32.

12.TOTU, E. E., VOICILA, E., PISTRITU, V., NECHIFOR, G., CRISTACHE, C. M., Rev. Chim.(Bucharest), 69, no.1, 2018, p. 155.

13.SETVIN, M., HAO, X., DANIEL, B., PAVELEC, J., NOVOTNY, Z., PARKINSON, G., S., SCHMID, M., KRESSE, G., FRANCHINI, C., DIEBOLD, U., Angew Chem Int Ed Engl, 53, 2014, p. 4714.

14.SHEN, J., WANG, H., ZHOU, Y., YE, N., LI, G., WANG, L., RSC Advances, 2, 2012, p. 9173.

15.STAGI, L., CARBONARO, C., M., CORPINO, R., CHIRIU, D., RICCl, P., C., Phys. Status Solidi B, 252, no. 1, 2015, p.124.

16.CARAUSU, E. M., CHECHERITA, L. E., STAMATIN, O., MANUC, D., Rev. Chim.(Bucharest), 67, no. 10, 2016, p. 2087.

17.WIEGAND, A., STUCKI, L., HOFFMANN, R., ATTIN, T., STAWARCZYK, B., Clin Oral Investig.,19, no.8, 2015, p. 2007.

18.TOTU, E. E., CRISTACHE, C. M., ISILDAK, I., YILDIRIM, R., BURLIBASA, M., NIGDE, M., BURLIBASA, L., Rev. Chim.(Bucharest), 69, no.5, 2018, p.1160.

Manuscript received: 26.08 .2018 